\title{
Improvements of InGaN/GaN quantum-well interfaces and radiative efficiency with InN interfacial layers
}

\author{
Yung-Chen Cheng, Cheng-Ming Wu, Meng-Kuo Chen, C. C. Yang, ${ }^{\text {a) }}$ and Zhe-Chuan Feng \\ Graduate Institute of Electro-Optical Engineering, Graduate Institute of Electronics Engineering, and \\ Department of Electrical Engineering, National Taiwan University, 1, Roosevelt Road, Sec. 4, Taipei, Taiwan, \\ Republic of China \\ Gang Alan Li \\ ShenZhen FangDa GuoKe Optronics Technical Co. Ltd., ShenZhen City, China \\ Jer-Ren Yang \\ Department of Material Science and Engineering, National Taiwan University, Taipei, Taiwan, \\ Republic of China \\ Andreas Rosenauer \\ Laboratorium für Elektronenmikroskopie, Universität Karlsruhe, 76128 Karlsruhe, Germany \\ Kung-Je Ma \\ Department of Mechanical Engineering, Chung Hua University, Hsinchu, Taiwan, Republic of China
}

(Received 3 February 2004; accepted 10 May 2004; published online 17 June 2004)

\begin{abstract}
The optical properties and nanostructures of two InGaN/GaN quantum-well (QW) samples of slightly different structures are compared. In one of the samples, InN interfacial layers of a few monolayers are added to the structure between wells and barriers for improving the QW interface quality. Compared with the standard barrier-doped QW sample, the addition of the InN interfacial layers does improve the QW interface quality and hence the photon emission efficiency. The strain state analysis images show the high contrast between the clear QW interface in the sample with InN layers and the diffusive QW boundaries in the reference sample. The detection-energy-dependent photoluminescence excitation data reveal the consistent results. (C) 2004 American Institute of Physics. [DOI: 10.1063/1.1767603]
\end{abstract}

Due to the large lattice mismatch between $\mathrm{InN}$ and $\mathrm{GaN}$, it is usually difficult to grow high-quality $\mathrm{InGaN} / \mathrm{GaN}$ quantum wells $(\mathrm{QWs}) .^{1-4}$ In particular, the diffusive interface between a well and a barrier usually leads to poor quantum confinement and hence lower photon emission efficiency. Also, with the diffusive QW structures, it becomes difficult to predict and hence design the emission wavelength for practical light-emitting devices. Furthermore, the low-quality interface may result in defect states and hence deteriorate the performance of such a light-emitting device. ${ }^{5,6}$ The diffusive QW interfaces can be attributed to the migrations of indium and gallium atoms through the spinodal decomposition process and other strain-induced effects. Although such processes can result in the formation of quantum dot-like clusters for localizing carriers and hence effective recombination, ${ }^{7-9}$ defects like dislocations may exist at the boundaries of the clusters, ${ }^{10}$ leading to the degradation of photon emission efficiency. Therefore, the improvement of QW quality, particularly the interfacial quality, is important for the development of the related light-emitting devices.

In this letter, we compare the photoluminescence (PL) and electroluminescence (EL) properties of two $\mathrm{InGaN} / \mathrm{GaN}$ QW samples with different well/barrier interface structures. $\mathrm{InN}$ thin layers of about $1 \mathrm{~nm}$ in thickness are inserted between a well and the neighboring barriers for improving the interfacial quality. With optical characterization and nanostructure analysis [strain state analysis (SSA)], it is found that with the InN interfacial layers, the photon emission ef-

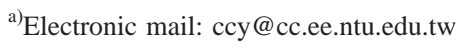

ficiency is improved when compared with a standard InGaN/GaN QW sample of barrier doping.

The two InGaN/GaN QW samples were grown on $c$-plane sapphire with metal organic chemical vapor deposition. After a $4 \mu \mathrm{m}$ Si-doped $\mathrm{GaN}\left(4 \times 10^{18} \mathrm{~cm}^{-3}\right.$ in doping concentration) layer, eight periods of QWs were grown with $2.5 \mathrm{~nm}$ in well width and $13.5 \mathrm{~nm}$ in barrier width. The barriers were Si-doped with $5 \times 10^{17} \mathrm{~cm}^{-3}$ in doping concentration. The nominal indium content of the InGaN wells was estimated to be $20 \%$. On top of the QW layers, a $150 \mathrm{~nm}$ $p$-GaN layer $\left(3 \times 10^{17} \mathrm{~cm}^{-3}\right.$ in carrier concentration $)$ was grown for preparing the Ohmic contact. The above-described sample is designated as sample B. In sample A, a Si-doped $\mathrm{InN}$ thin layer of about $1 \mathrm{~nm}$ in thickness was inserted into the sample between a well and its neighboring barrier on either side. The growth temperatures for all layers were the same at $735{ }^{\circ} \mathrm{C}$ with very low growth rates and high V/III ratios. The injection currents in the EL measurements for samples A and B were 1.1 and $1.5 \mathrm{~mA}$, respectively. The electric current levels were designated right above the threshold values for effective output detection. The comparison of injection current shows that sample A is superior to sample B in EL performance.

Figure 1 shows the PL and EL spectra of the two samples at several temperatures. In the EL spectra, two major peaks can be observed, particularly at low temperatures. In either sample, the major part of EL intensity shifts from the high-energy peak to the lower one as temperature increases. ${ }^{11}$ The redshift trend with increasing temperature, shown in Fig. 1, is opposite to the previously reported blue- 


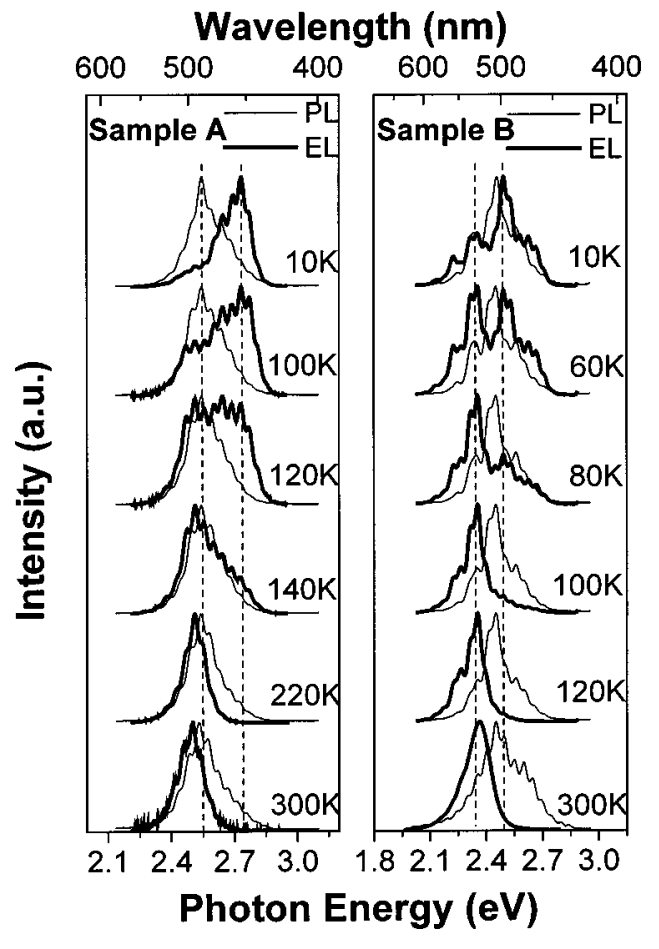

FIG. 1. PL (thin lines) and EL (thick lines) spectra of the two samples at various temperatures. The two vertical dashed lines roughly indicate the positions of the two peaks.

shift behavior. ${ }^{12}$ Figure 2 summarizes the PL and EL spectral peak positions of the two samples. In sample B, the highenergy peak exists up to $80 \mathrm{~K}$. In sample $\mathrm{A}$, the high-energy peak exists up to $120 \mathrm{~K}$ and the low-energy one cannot be identified below this temperature. In both samples, PL spectral peaks lie between the two EL peaks in the whole temperature range. The high-energy peaks in the EL spectra can be attributed to the carrier activities in the QW energy states (or called free-carrier states). The low-energy peaks correspond to those around the potential minima of clusters (or called localized states) within the QWs. ${ }^{13,14}$ The localized states are also responsible for the PL emission. As mentioned earlier, in either sample, the major EL emission intensity shifts from the high-energy peak to the low-energy one as temperature increases. The uniquely existent low-energy peak in the high-temperature range originates from the liquidation of thermalized carriers among the QW and localized states. In this situation, more carriers may exist in the localized states, leading to the dominance of the low-energy EL peak.

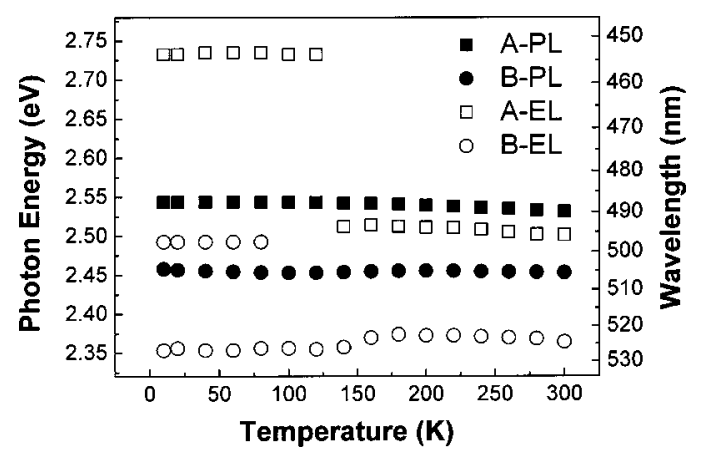

FIG. 2. PL and EL spectral peak positions of the two samples as functions of temperature. The two peaks are shown whenever they can be clearly calibrated.

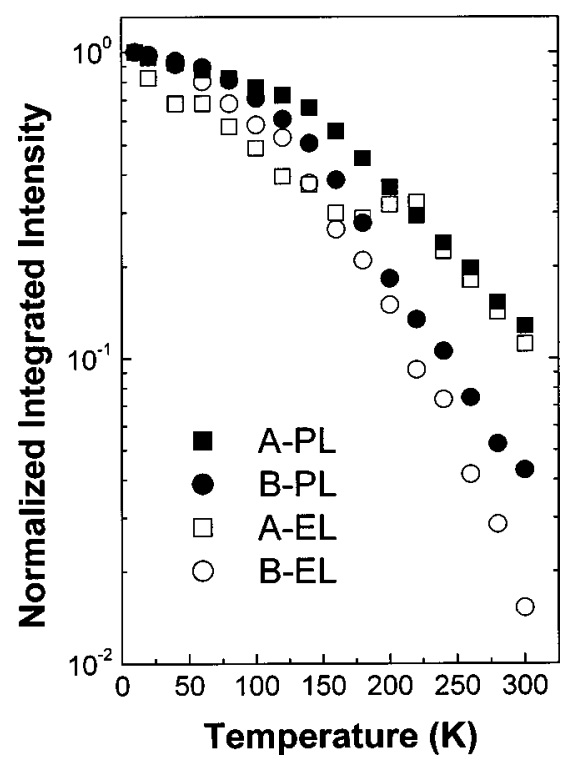

FIG. 3. Normalized integrated intensities of PL and EL of the two samples as functions of temperature.

In Fig. 2, one can see that the localized-state-related EL is redshifted from PL in both samples. This trend can be attributed to the application of forward bias voltage in current injection for EL measurements. With the forward bias, the originally existing potential slope (due to the piezoelectric field) is further tilted, leading to a stronger quantumconfined Stark effect (QCSE) and hence the redshifts. The QCSE can be partially compensated by the carrier screening effect, resulting in a reduced potential tilt. From the results in Fig. 2, one can speculate that the effective increment of potential tilt due to current injection in sample $\mathrm{A}$ is smaller. Hence, the differences between the localized-state-related EL and PL peaks of sample A are smaller. The different redshift extents of the low-energy EL peak with respect to the PL peak between the two samples is attributed to their different strain distributions. In sample B, the formation of cluster structures can release the strain energy. Hence, the piezoelectric field or QCSE in this sample is relatively weaker. With a weaker QCSE in sample B, the effect of the applied forward bias voltage seems to be stronger.

Figure 3 shows the normalized integrated intensities of PL and EL as functions of temperature in the two samples. The decay slope of such a curve represents the radiative efficiency. Here, one can see that in either PL or EL measurement, the radiative efficiency of sample A is significantly higher than that of sample B. The addition of the InN interfacial layer can improve the photon emission efficiency.

Figure 4 shows the PL and detection-energy-dependent photoluminescence excitation (DEDPLE) spectra at $10 \mathrm{~K}$ of the two samples. The DEDPLE spectra are normalized at $3.5 \mathrm{eV}$, which corresponds to the GaN barrier band gap. Compared with sample A, the InGaN absorption features are much stronger, relative to the GaN peak, in sample B. In particular, with a high detection energy level at $2.583 \mathrm{eV}$, the InGaN absorption is even stronger than $\mathrm{GaN}$ in sample B. Such features imply that the QW interfaces in sample B are unclear. In other words, stronger InGaN composition fluctuations exist in sample B. Also, certain regions of InGaN compositions close to GaN exist in this sample. The plenty InGaN energy states result in strong absorption for PL 


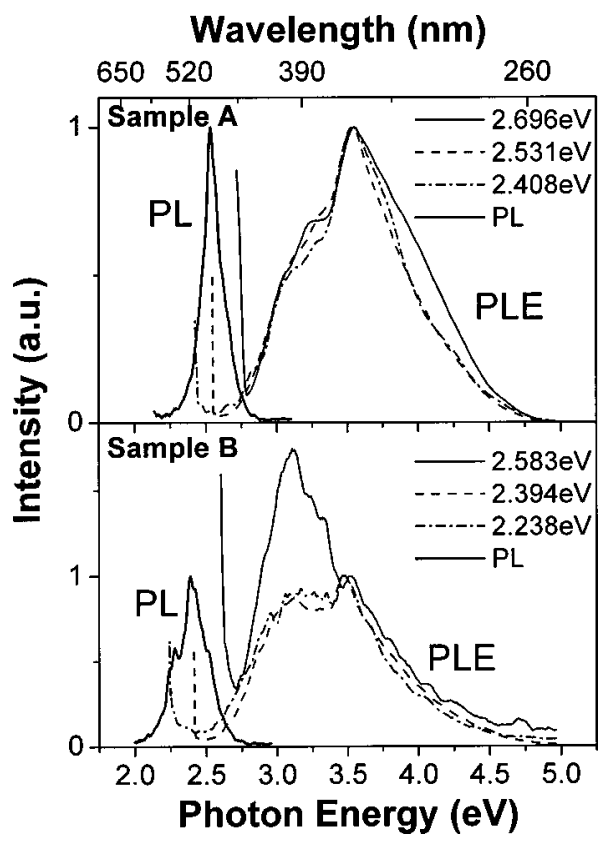

FIG. 4. DEDPLE and PL spectra of the two samples at $10 \mathrm{~K}$. The PLE spectra are normalized at $3.5 \mathrm{eV}$, the $\mathrm{GaN}$ band gap.

emission at the deeply localized states. The comparison between samples A and B in Fig. 4 implies again that the QW interfacial quality of sample $\mathrm{A}$ is higher.

To confirm the quality of QW interface, we performed high-resolution transmission electron microscopy observation. With the two-beam interference data, we could conduct the SSA for composition distribution images. ${ }^{15}$ Figures 5 and 6 show the typical SSA images of samples A and B, respectively. In these SSA images, line scans were conducted along the shown white lines. Here, the line scan values 1 and 1.1, respectively, represent indium compositions of 0 and $60 \%$. Different colors stand for various ranges of indium composition, as shown in the legends. As shown in Fig. 5, the shape of the $\mathrm{QW}$ is quite clear although its thickness varies slightly. Its boundaries are quite abrupt although indium composition varies in space within the well. In particular, the upper interface in the image is quite clear-cut. Such features can also be clearly seen in the line scan plots. The well-defined QW layer in sample A is particularly clear when it is compared with the diffusive nanostructure of sample B, as shown in Fig. 6. Here, one can see the strongly clustering structure

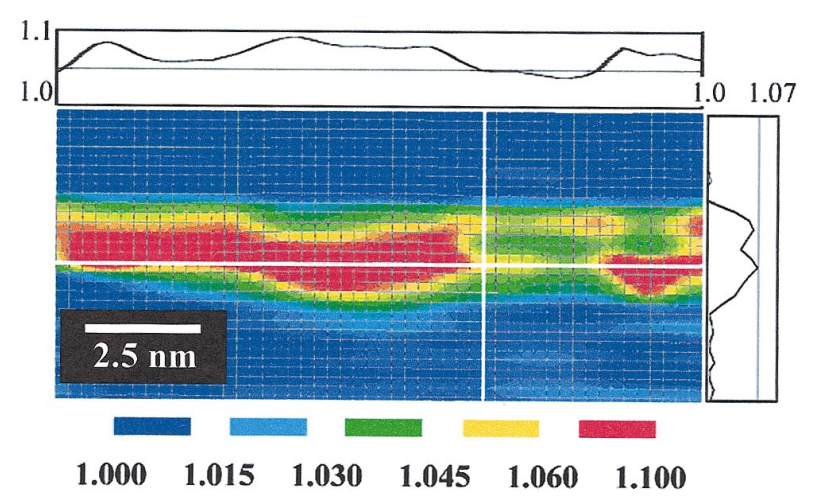

FIG. 5. (Color) A typical strain-state analysis (SSA) image of sample A.

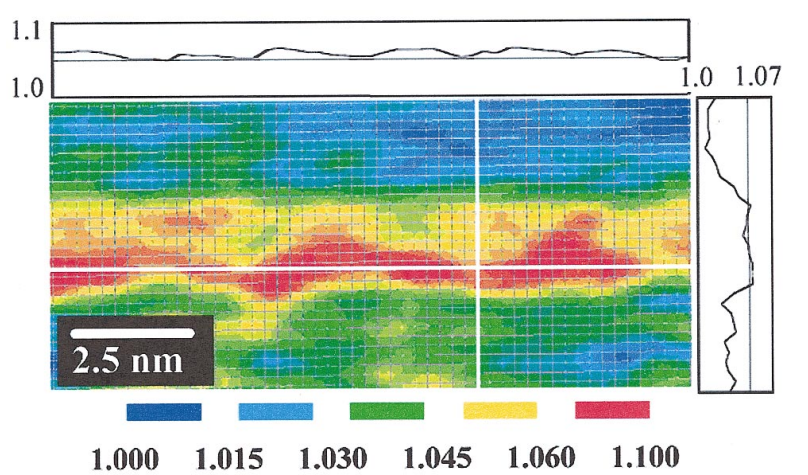

FIG. 6. (Color) A typical SSA image of sample B.

around the designated QW layer although the QW shape is still recognizable. The strong indium composition fluctuation shown here confirms the strong InGaN absorption features in Fig. 4. It is believed that the higher QW interface quality shown in Fig. 5 results in the higher photon emission efficiency of sample A, as indicated in Fig. 3.

In summary, we have compared two InGaN/GaN QW samples in optical property and nanostructure. In one of the samples, InN interfacial layers were placed between wells and barriers for improving the QW interface quality. Compared with the standard barrier-doped QW sample, the addition of the InN interfacial layers did improve the QW interface quality and hence the photon emission efficiency.

This research was supported by National Science Council, The Republic of China, under Grant Nos. of NSC 922210-M-002-006 and NSC 92-2215-E-002-010, and by US Air Force under Contract No. AOARD-02-4052.

${ }^{1}$ K. Watanabe, J. R. Yang, N. Nakanishi, K. Inoke, and M. Shiojiri, Appl. Phys. Lett. 80, 761 (2002).

${ }^{2}$ H. K. Cho, J. Y. Lee, N. Sharma, C. J. Humphreys, G. M. Yang, C. S. Kim, J. H. Song, and P. W. Yu, Appl. Phys. Lett. 81, 3102 (2002).

${ }^{3}$ Y. S. Lin, K. J. Ma, C. Hsu, Y. Y. Chung, C. W. Liu, S. W. Feng, Y. C. Cheng, M. H. Mao, C. C. Yang, H. W. Chuang, C. T. Kuo, J. S. Tsang, and T. E. Weirich, Appl. Phys. Lett. 80, 2571 (2002).

${ }^{4}$ Y. S. Lin, K. J. Ma, C. Hsu, S. W. Feng, Y. C. Cheng, C. C. Liao, C. C. Yang, C. C. Chuo, C. M. Lee, and J. I. Chyi, Appl. Phys. Lett. 77, 2988 (2000).

${ }^{5}$ M. Hao, H. Ishikawa, T. Egawa, C. L. Shao, and T. Jimbo, Appl. Phys. Lett. 82, 4702 (2003).

${ }^{6}$ D. I. Florescu, S. M. Ting, J. C. Ramer, D. S. Lee, V. N Merai, A. Parkeh, D. Lu, E. A. Armour, and L. Chernyak, Appl. Phys. Lett. 83, 33 (2003).

${ }^{7}$ S. W. Feng, E. C. Lin, T. Y. Tang, Y. C. Cheng, H. C. Wang, C. C. Yang, K. J. Ma, C. H. Shen, L. C. Chen, K. H. Kim, J. Y. Lin, and H. X. Jiang, Appl. Phys. Lett. 83, 3906 (2003).

${ }^{8}$ Y. C. Cheng, C. H. Tseng, C. Hsu, K. J. Ma, S. W. Feng, E. C. Lin, C. C. Yang, and J. I. Chyi, J. Electron. Mater. 32, 375 (2003).

${ }^{9}$ S. Chichibu, K. Wada, and S. Nakamura, Appl. Phys. Lett. 71, 2346 (1997).

${ }^{10}$ Y. S. Lin, C. C. Yan, C. Hsu, K. J. Ma, Y. Y. Chung, S. W. Feng, Y. C. Cheng, E. C. Lin, C. C. Yang, C. T Kuo, and J. S. Tsang, J. Cryst. Growth 252, 107 (2003).

${ }^{11}$ C. M. Lee, C. C. Chuo, J. F. Dai, X. F. Zheng, and J. I. Chyi, J. Appl. Phys. 89, 6554 (2001).

${ }^{12}$ X. A. Cao, S. F. LeBoeuf, L. B. Rowland, C. H. Yan, and H. Liu, Appl. Phys. Lett. 82, 3614 (2003).

${ }^{13}$ C. C. Liao, S. W. Feng, C. C. Yang, Y. S. Lin, K. J. Ma, C. C. Chuo, C. M. Lee, and J. I. Chyi, Appl. Phys. Lett. 76, 318 (2000).

${ }^{14}$ T. J. Schmidt, Y. H. Cho, G. H. Gainer, J. J. Song, S. Keller, U. K. Mishra, and S. P. DenBaars, Appl. Phys. Lett. 73, 560 (1998).

${ }^{15}$ D. Gerthsen, B. Neubauer, A. Rosenauer, T. Stephan, H. Kalt, O. Schon, and M. Heuken, Appl. Phys. Lett. 69, 2701 (1996). 